\title{
The existence and extent of spatial working memory ability in honeybees
}

\author{
MICHAEL F. BROWN, JONATHAN A. MOORE, \\ CATHERINE H. BROWN, and KRISTEN D. LANGHELD \\ Villanova University, Villanova, Pennsylvania
}

\begin{abstract}
Honeybees foraged from six locations, each of which was baited with sugar solution prior to each experimental trial. Under a variety of conditions, bees exhibited a small but reliable tendency to avoid revisits to locations that they had visited earlier during the experimental trial. These results replicate those of Brown and Demas (1994), who concluded that bees use working memory to discriminate previously visited locations from those not yet visited. The present experiments included procedures that allowed alternatives to this explanation to be more completely ruled out. The extent of spatial working memory performance exhibited by honeybees in these experiments appears to be limited by a process other than working memory capacity, perhaps the ability of bees to discriminate among several locations in close proximity to one another.
\end{abstract}

A distinction fundamental to our understanding of human and animal memory systems is that between reference memory and working memory (see, e.g., Baddeley, 1986; Honig, 1978). Information that remains more or less stable over time is thought to be stored in a largecapacity, long-duration reference memory system, whereas a relatively small-capacity and often short-duration system is used to store information that changes in content often or is only temporarily useful. In humans, working memory is generally considered to be the cognitive structure in which information is stored while active cognitive processes operate on that information (Baddeley, 1986).

A large number of studies have examined working memory in a variety of vertebrate animals, primarily using pigeons in the matching-to-sample procedure (e.g., Roberts \& Grant, 1974) and rats in the radial-arm maze procedure (e.g., Olton \& Samuelson, 1976). In both cases, one or more conditional cue(s) must be stored in memory to bridge a temporal gap prior to the critical choice response(s). In matching-to-sample, the identity of the sample determines the test stimulus to which the subject must later respond for reinforcement. In the radial-arm maze, the identity of previously visited spatial locations determines the locations that will be baited later in the trial. The fact that these cues change from trial to trial requires a flexible, dynamic system for the temporary storage of information (i.e., working memory).

Brown and Demas (1994) recently provided initial evidence for working memory in honeybees using a proce-

This research was supported by National Science Foundation (NSF) Grant IBN-9404120. The participation of Kristen Langheld was made possible by an NSF Research Experience for Undergraduates award. We thank Jessica Lease for assistance conducting Experiment 1. Correspondence should be addressed to M. F. Brown, Department of Psychology, Villanova University, Villanova, PA 19085 (e-mall: mbrown (a), emall.vill.edu) dure that is analogous to the radial-arm maze procedure used with rats and other vertebrates. There is an abundance of evidence for various forms of learning and memory in honeybees and other invertebrates (for recent reviews, see Abramson, 1994; Bitterman, 1996; Menzel, 1990; Papaj \& Lewis, 1993). However, Brown and Demas's data are the first to suggest that honeybees possess a flexible, dynamic, multiple-item memory system, corresponding to the working memory that has been studied in humans and other vertebrates.

If honeybees do in fact possess a memory system analogous to, or similar to, vertebrate working memory, this fact would have wide-ranging implications for our understanding of working memory. For example, working memory in vertebrates has been associated with hippocampal and other brain structures that do not even exist in invertebrates. Thus, working memory (or a system analogous to working memory) can be implemented in a brain that is very different from those in which it has been studied so far.

Brown and Demas (1994; Demas \& Brown, 1995) reported a total of four experiments in which honeybees foraged for small drops of sucrose solution from a matrix of six closely spaced locations. Bees depleted each location of sucrose during the first visit to the location. Thus, just as in Olton's radial-arm maze problem (Olton \& Samuelson, 1976), the contingencies of reinforcement encouraged one and only one visit to each location during each experimental trial. Brown and Demas used a Monte Carlo simulation to estimate the expected tendencies of the bees to revisit locations given their location-to-location transition probabilities. They found that the tendency of the bees to revisit locations was slightly but significantly lower than these estimates. Their experiments included controls for the possibility that a perceptual cue related to the presence of the sucrose solution allowed this avoidance of revisits and for the possibility that an odor trail 
left by the bees controlled choice. Thus, Brown and Demas inferred that the bees were using working memory to discriminate the locations already visited during an experimental trial from those not yet visited.

The present experiments address two issues related to this recent evidence for working memory in bees. The first stems from recent work by Burmeister, Couvillon, and Bitterman (1995), which questions Brown and Demas's (1994) conclusions on both empirical and theoretical grounds. The second is related to the magnitude of the effect reported by Brown and Demas. Their evidence for working memory was quite reliable, but small in magnitude relative to working memory effects that have been reported in vertebrates. This may mean that honeybees have only limited working memory ability. If so, it may be that the bee nervous system can support only limited working memory ability. Alternatively, it may be that the experimental procedures used by Brown and Demas did not result in an accurate assessment of the magnitude of bee working memory ability. Most of the work reported below was designed to investigate this possibility.

\section{EXPERIMENT 1}

Burmeister et al. (1995; see also Isnec, Couvillon, \& Bitterman, 1997; Ohyama, Couvillon, \& Bitterman, 1995) recently reported three experiments, using arrangements of three or four baited locations, in which they failed to find evidence of any tendency to avoid revisits to locations. They did find systematic biases in their bees to visit locations according to their spatial properties. Deviations from a priori estimates of chance levels of choice accuracy in their bees can be explained in terms of these biases. Although Burmeister et al. did not offer an explanation that would account for the discrepancy between their results and ours, they did argue that our experimental methods were "highly unsatisfactory" for several reasons. First, they correctly pointed out (just as we did) that odor trails can explain the results of our previous free-choice experiments (Brown \& Demas, 1994, Experiment 1; Demas \& Brown, 1995). That is, when the bee visits a location, it might leave an odor trace and use this trace as a perceptual cue during subsequent choices. However, such odor trails cannot explain the results of our previous forced-choice experiments (Brown \& Demas, 1994, Experiments 2 and 3). In those forced-choice experiments, the apparatus was switched with a fresh (and therefore odorless) one between the third of three forced choices and a free-choice phase of each experimental trial. The ability of bees to avoid revisits to the first three (forced) locations during these free choices was compared with the tendency to make such revisits predicted by a Monte Carlo simulation.

Second, Burmeister et al. (1995) pointed out that our previous experimental technique did not include the bees' feeding to repletion during each trial. They argued that this aspect of our technique could have resulted in trials being "given under different motivational conditions and at different points in the subject's foraging cycle, or, if the excluded subject went off to forage elsewhere, at widely different intertrial intervals" (p. 374). Although they did not specify how these differences could have produced the pattern of results we obtained, Burmeister et al. are correct that this factor could have affected our results. Finally, they pointed out that most of our experiments (all but Experiment 3 of Brown \& Demas, 1994) were conducted quite close to the hive, resulting in many bees other than the subject bee being attracted to the area of the experimental apparatus. To avoid interference by nonsubject bees, we enclosed our apparatus in a clear plastic container, including a lid that prevented the bees from flying higher than $14 \mathrm{~cm}$ above the surface of the apparatus. Burmeister et al. asserted that this restriction of subjects' flying room may have affected their behavior. It has also been suggested (by an anonymous reviewer of a grant proposal) that the Plexiglas lid used to restrict access to the apparatus may have filtered ultraviolet light that might have been involved in the use of spatial cues by bees.

Although the first of these three criticisms applies only to some of our previous experiments and it is not clear how the remaining two criticisms can explain the pattern of results we obtained, we believe that the criticisms raised by Burmeister et al. (1995) should be taken seriously. There is convincing evidence that an odor trail can control honeybee choice in a laboratory task somewhat similar to ours (Giurfa \& Núñez, 1992). There is also some evidence that this occurs during natural foraging (Corbet, Kerslake, Brown, \& Morland, 1984; Free \& Williams, 1983; Wetherwax, 1986). Thus, an additional test of the avoidance of revisits by honeybees that cannot be explained in terms of an odor trail is clearly important.

The experimental procedure of the first experiment in the present series was very similar to that used by Brown and Demas (1994, Experiment 1), but differed in ways suggested by the criticisms of Burmeister et al. (1995). First, a technique similar to that used by Burmeister et al. was used to prevent control of choices by an odor trail, in the context of a free-choice procedure. Specifically, the six target locations contained small, square dishes, with the drop of sucrose solution centered in the dish. Following the first visit to a target location, the dish was replaced with a dish containing a drop of water. Thus, any odor left behind by the bee during the first visit to a target location was removed.

Second, when the bee chose the target location containing the last drop of sucrose solution and began feeding on that drop, the drop was enlarged so that the bee fed to repletion. This was intended to encourage the bees to return to the hive between trials, thereby increasing the match between the conditions to which the bee was exposed and the trial structure intended by the experimental design. Finally, we conducted the present experiment relatively far from the hive, inside a laboratory. As a result, very few bees that had not been intentionally recruited to the experiment entered the area of the appara- 
tus. Therefore, there was no need to cover the apparatus and restrict the flying area of the bees, as we did in our earlier experiments.

\section{Method}

Subjects. The subjects were 14 honeybees (Apis mellifera ligustica) from a full-sized hive located inside a campus building, with passage outside via a tunnel leading from the hive through a window. The hive was three floors above the laboratory where data were collected.

Apparatus. The apparatus consisted of a $2 \times 3$ matrix of target locations on a $28.0 \times 28.0 \times 6 \mathrm{~mm}$ (thick) plywood surface. If the matrix is considered to be two columns of three target locations each, then the two columns of locations were separated by $17.3 \mathrm{~cm}$ (center to center). The locations forming the ends of the columns were separated by $22.7 \mathrm{~cm}$, with the middle location of each column being $9.8 \mathrm{~cm}$ from one end of the column and the middle hole of the other column being the same distance from the other end of the other column. The plywood surface was painted violet. Each target location was defined by the presence of a $4.4-\mathrm{cm}$-square, $0.95-\mathrm{cm}$ deep, translucent polystyrene weighing boat (referred to below as a "dish"; Cole-Parmer No. H-01017-05), placed inside a white, porcelain crucible $(7 \mathrm{~cm}$ in diameter and $1.2 \mathrm{~cm}$ deep). A red adhesive dot $(1.3 \mathrm{~cm}$ in diameter) on the surface of each crucible and visible through the bottom of the dish was intended as a visual cue defining the point on each target location where drops of sucrose solution (or water) were placed. The dish/crucible units defining each target location could be easily removed from the apparatus surface (see Procedure). Six landmarks, consıstıng of differently colored and shaped children's blocks, were glued to the surface of the apparatus, one in close proximity to each of the target locations. The apparatus was located on the laboratory window ledge, with the window open. Dishes were baited with $50 \%(\mathrm{v} / \mathrm{v})$ sugar solution at room temperature, using a microsyringe.

Procedure. Subjects were collected by netting them as they left the entrance to the hive. They were taken in individual vials to the laboratory, and these vials were placed (open end down) over a dish at a target location on the apparatus, which had been baited with a large drop of sugar solution. Movement of the bee to the bottom of the vial (and therefore the dish) was sometimes facilitated by placing an opaque cover over part of the vial. The bee was allowed to feed from the dish. After feeding to repletion, it flew out of the laboratory through the window. If a bee returned to the apparatus, it was considered to be a subject in the experiment and was marked using a spot of Testor's model paint on the thorax or abdomen.

Following successful recruitment to the apparatus, each trial began by placing a freshly baited dish (baited with a 4- to 5- $\mu$ l drop of solution in its center) at each of the six target locations. When the experimenter detected the bee approaching the window, a camcorder was turned on to record the subject's behavior. The few nonsubject bees that entered the laboratory were prevented from interfering with the experiment by chasing them away or capturıng them.

A choice was defined when any part of the subject bee touched a dish. This was virtually always followed by a full landing and extension of the probosc1s onto the surface of the dish. When a bee left a dish following the initial visit, the dish was removed and replaced with one containing a fresh 4- to 5- $\mu$ l drop of tap water. The bees spent several seconds imbibing the sucrose solution following a correct choice, but immediately rejected the water drop following an incorrect choice. Because of this, we did not attempt to replace dishes containing water drops with fresh dishes contaıning water drops following an incorrect choice. The speed with which most bees moved from dish to dish toward the end of a trial (when many dishes contained water drops) did not allow us to unfalingly replace dishes before the bee made its next choice. Because of the time taken to imbibe sucrose solution during choices made early in the choice sequence, however, we were able to replace dishes following the initial visit to a target location between the time the bee left that location and the time it left the location of its next choice. The sequence of choices made by the bee was recorded, using the videotape record to resolve any ambiguous cases.

When a bee chose the target location containing the last remaining sugar drop, a syringe was used to enlarge the drop so that it was larger than could be imbibed by the bee. This resulted in the bee imbibing a large amount of solution and then flying out the window. A trial was considered complete when the bee flew out the window and did not return for at least $3 \mathrm{~min}$. Typically, the bee disappeared for 10-15 min following a trial and then returned. In the meantime, the apparatus was prepared for the next trial by replacing all six dishes with freshly baited ones. Trials continued until a bee had completed 20 trials, or until the bee ceased returning for additional trials.

Beginning with the 4 th bee that participated in the experiment, if a bee completed all 20 trials, the procedure described above was followed by five trials of a perceptual cue probe procedure, designed to ensure that bees could not discriminate drops containing sugar from water-only drops. During each of these five trials, a randomly selected set of three locations was baited with sugar solution and the remaining three were baited with water drops. Bees were allowed to choose from among the six locations until all six had been visited. Dishes were not replaced following a visit.

\section{Results}

The 14 subjects participated in a mean of 17.2 trials with six or more choices. Bees visited all six target locations during a mean of $84.0 \%$ of these trials. For purposes of data analysis, trials were considered to be structured into two blocks of 10 trials each. Only trials meeting a criterion of including at least six visits were further analyzed. If a bee did not participate in at least five such trials during a trial block, its data from that block were not included. This was the case for 4 bees during the second trial block. These 4 bees did not complete the planned regimen of 20 trials because the bee ceased visiting the apparatus for unknown reasons.

Two measures of choice accuracy during the first six visits were determined for each bee. The first measure is the number of visits that were to correct (previously unvisited) target locations. Immediate revisits (i.e., revisiting a location without an intervening visit to another location) were not counted as visits because of a potential artifact. Specifically, we could not be sure that our manipulation of dishes following initial visits (i.e., removing the old dish and replacing it with a fresh dish containing a water drop) did not have an effect on the tendency to immediately revisit. Certainly, the bees did not have this option while the manipulation was being carried out. So this behavior was considered undefined both in the empirical results and in our estimate of chance. This strategy eliminates any cuing effect that our manipulation of the apparatus to replace target locations might have had.

A second measure of choice accuracy during the first six visits was used because of the fact that a fresh dish replaced a visited one only following the initial visit to a target location. It is possible that bees may have avoided visiting a target location for a third time because of odor left there during the second visit. Restricting the analyses to choices made during the first six choices reduces any 
influence this possibility may have had. Nevertheless, it was desirable to develop a measure that eliminated any influence of odor left following the second (or subsequent) visits to target locations. This measure is the mean number of target locations revisited (one or more times) during the first six visits. Thus, a target location that is visited three times during the first six choices counts as being revisited in exactly the same manner as a target location that is visited twice. This measure therefore eliminates the small number of cases in which a bee visited the same location for a third (or more) time.

These two measures of choice accuracy were compared to estimates of chance performance. Appropriate estimates of chance in this task must take into account the nonrandom location-to-location transition biases that bees demonstrate (Brown \& Demas, 1994; Burmeister et al., 1995). In our apparatus, for example, bees tend to travel from one location to a location in relatively close proximity. This and other transition tendencies affect the choice accuracy of the bees in a manner assumed to be independent of working memory ability. To control for these effects, Brown and Demas (1994) used Monte Carlo simulations of bee choice behavior that incorporated the location-to-location transition probabilities of individual bees during individual trial blocks. This same technique was used to provide comparisons for the empirical results found in the present experiment. First, a transition probability matrix was constructed for each bee during each of the two trial blocks. Given that the bee was in each of the six locations (or had just started a trial and not yet visited a location), the probability of moving from that location (or from entering a trial) to each of the six locations was determined. The overall transition probability matrix is shown in Table 1 . The transition probability matrices of individual bees for each block of trials were used in Monte Carlo simulations that chose locations using the same transition probabilities as each bee. Any tendency to avoid revisits to locations beyond what is shown by these simulations cannot be accounted for by systematicity in the location-to-location movement patterns of the bees.

Table 1

Overall Transition Probability Matrix for Choices Made in Experiment 1

\begin{tabular}{ccccccr}
\hline & \multicolumn{7}{c}{ Destination Location } \\
\cline { 2 - 7 } Previous Location & \multicolumn{1}{c}{1} & \multicolumn{1}{c}{2} & \multicolumn{1}{c}{3} & 4 & 5 & \multicolumn{1}{c}{6} \\
\hline Begin trial & 6.6 & 14.9 & 7.5 & 33.2 & 17.8 & 19.9 \\
Location 1 & $*$ & 41.9 & 4.5 & 33.6 & 14.5 & 5.5 \\
Location 2 & 27.6 & $*$ & 24.8 & 12.6 & 21.7 & 13.3 \\
Location 3 & 5.2 & 39.8 & $*$ & 3.6 & 18.1 & 33.2 \\
Location 4 & 34.8 & 14.3 & 5.1 & $*$ & 38.3 & 7.5 \\
Location 5 & 6.7 & 16.9 & 6.7 & 33.0 & $*$ & 36.7 \\
Location 6 & 1.6 & 6.8 & 49.2 & 6.3 & 36.1 & $*$ \\
\hline
\end{tabular}

Note-Each row shows the percentage of transitions (location-to-location movements) that were to each of the six locations, given the previous location of the bee (either the location chosen last or initial entry into the apparatus for a new trial). Data are collapsed across bees and trial blocks. * Immediate returns to locations are ignored in the analysis. The locations are numbered as two rows of three locations each (e.g., Location 2 is in between Locations 1 and 3, and is also adjacent to Location 5).
The algorithm used to implement the Monte Carlo simulations chose from among six alternatives using the location-to-location transition probabilities of individual subjects during individual trial blocks until all six had been chosen. The estimates produced are based on 1,000 simulations using each bee's transition matrix during each of the two trial blocks. The data produced by these Monte Carlo simulations were analyzed in terms of the same two measures of choice accuracy described above for the empirical data. Thus, like the empirical measures of performance, these estimates did not include any immediate revisits to locations.

The top panel of Figure 1 shows the mean number of correct choices made during the first six choices of each trial made by the bees and by the corresponding Monte Carlo simulations. Because 4 bees did not contribute to the data of Block 2, and it is possible that this subject loss was nonrandom, data for the two trial blocks were evaluated separately. The bees made more correct choices than estimated by the simulations during both Block $1[t(13)=$ $3.88]$ and Block $2[t(9)=3.84]$ (all statistical decisions reported in this paper were made using a rejection criterion of $p<.05$ ). The bottom panel of Figure 1 shows the number of target locations revisited (one or more times) during each of the two trial blocks. The bees revisited fewer locations than estimated by the simulations during both Block $1[t(13)=2.68]$ and Block $2[t(9)=2.65]$.

Seven bees participated in the perceptual cue probe trials that followed the primary experimental trials. For each trial, the ordinal rank of the initial visit to each of the six locations was determined (the first location visited was given a rank of 1 , the second a rank of 2 , etc.). The mean ordinal ranks of the locations baited with sugar solution and water were determined for each bee. The mean (over bees) ordinal ranks were 3.49 and 3.51 for the locations baited with sugar and water, respectively $[t(6)=.08]$.

\section{Discussion}

These results confirm the finding of Brown and Demas (1994) that bees avoid revisits to locations that have recently been depleted of sucrose solution. Because the estimates of chance to which choice behavior is compared control for systematicity in the location-to-location transition probabilities, this tendency cannot be attributed to consistent movement patterns or biases in the bees' behavior. The avoidance of revisits cannot be explained by an ability to discriminate the presence of sugar solution, as shown by the results of the perceptual cue probe trials. Rather, information about the identity of previously visited locations has some control over choices. The experimental and analytic techniques used in the present experiment rule out the possibility that this control is based on a physical trace left by the bee during previous visits, such as an odor trail. Thus, this tendency is best attributed to memories of previous visits. Because the identity of previously visited locations changes both within each trial and from one trial to the next, the memory involved is best characterized as a form of working memory, analogous 

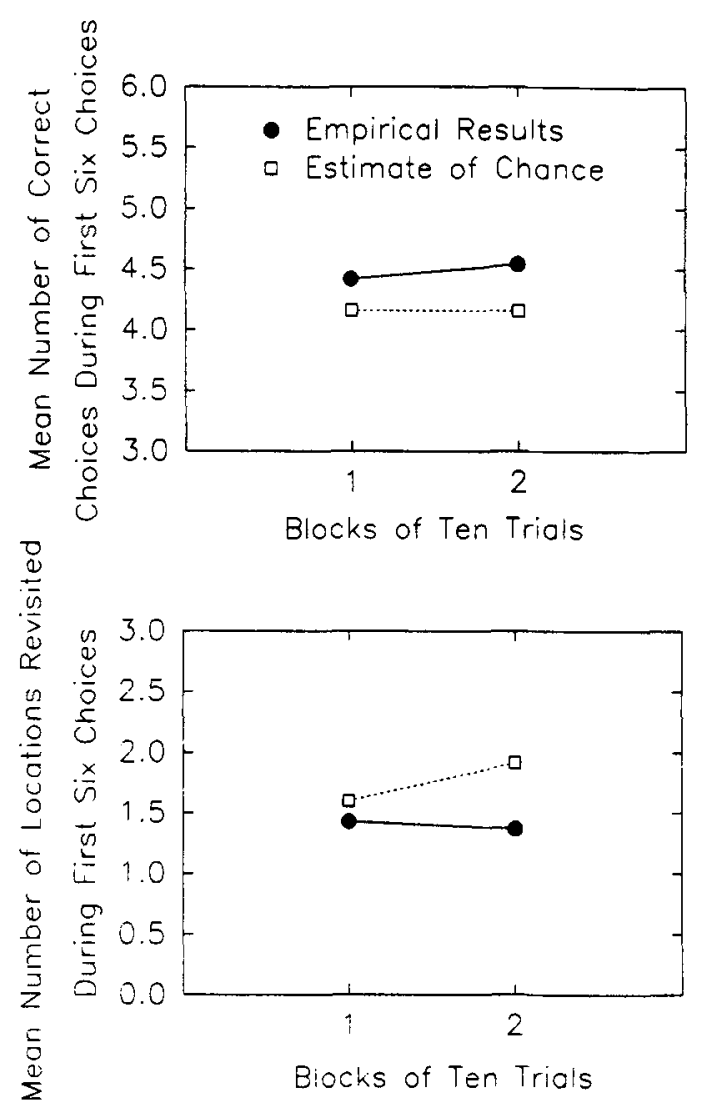

Figure 1. Mean values for two measures of choice accuracy of the bees and Monte Carlo simulations during the first six choices of trials in Experiment 1 . The measures are the number of correct choices made (top panel) and the number of target locations that were revisited one or more times (bottom panel). Data are shown separately for the two blocks of trials.

in function to that known to be involved in vertebrate delayed discrimination performance (see, e.g., Honig, 1978).

\section{EXPERIMENT 2}

The magnitude of the effects found in Experiment 1 and in our earlier experiments (Brown \& Demas, 1994; Demas \& Brown, 1995) appears rather small and suggests that the extent of spatial working memory ability in bees is correspondingly small. However, we cannot know whether the low level of working memory performance reflects a fundamental limitation in the working memory ability of bees or our failure to allow bees to express the full extent of their working memory ability. The failure of Burmeister et al. (1995) to find any tendency to avoid revisits is consistent with the possibility that bees possess limited working memory abilities.

Both from a theoretical and practical perspective, it is important to study conditions that might support higher levels of working memory performance in bees. Examination of the properties of this memory system will require that manipulations be used that modulate the level of working memory performance. This can be done only if there are conditions that support moderate to high levels of working memory performance. In addition, determination of the level of working memory performance that can be supported by the bee nervous system may be critical for furthering a comparative analysis of working memory.

The present experiment examined performance in a procedure very similar to that of Brown and Demas (1994; Experiment 1) and to that of the present Experiment 1. In three subexperiments, three different apparatuses were used that we thought might produce relatively higher levels of working memory performance. The first of these (Experiment 2A) was based on the possibility that bees would discriminate among six baited locations more effectively if the locations were separated in space more than they have been in our earlier experiments. Thus, in this experiment the baited locations were separated by $35 \mathrm{~cm}$ rather than by $10-15 \mathrm{~cm}$, as has been the case in our earlier experiments. If a limitation on working memory performance was produced in the earlier experiments by the difficulty of discriminating among locations because of their proximity, then higher levels of working memory ability might be found under these conditions.

Experiment 2B was motivated by our knowledge that, in rats, spatial working memory performance is increased when a relatively large amount of time or effort is required to visit each baited location (Brown \& Huggins, 1993; Brown \& Lesniak-Karpiak, 1993). This was implemented in bees by requiring that the bees crawl through a tube to reach each of six baited locations.

Experiment 2C involved a radial-arm maze (Olton \& Samuelson, 1976) with six arms. The radial-arm maze has been shown to support very high levels of spatial working memory performance in a variety of vertebrate species, including rats (see, e.g., Olton \& Samuelson, 1976), pigeons (Roberts \& Van Veldhuizen, 1985), and corvids (Kamil, Balda, \& Olson, 1994). Although the apparatuses used in our earlier experiments were inspired by the radial-arm maze and preserved some of its properties, it is possible that other properties of the radial-arm maze are critical for its ability to support high levels of performance. In particular, animals choose locations from a central location in the radial-arm maze, and at least one theory of spatial choice implies that this common choice point is important (Brown, 1993). Thus, in Experiment $2 \mathrm{C}$ bees visited six locations at the ends of arms of a radial-arm maze. The maze arms and central arena were enclosed with mesh.

\section{Method}

Subjects. The subjects in these experiments were from the same hive as those used in Experiment 1. They were netted as they left the hive and taken in plastic vials to the laboratory for the recruitment procedure (see below). Twenty, 20, and 16 subjects participated in Experiments $2 \mathrm{~A}, 2 \mathrm{~B}$, and $2 \mathrm{C}$, respectively.

Apparatus. The apparatuses used in these experiments are shown in Figure 2. The apparatus used in Experiment 2A (Figure 2, top panel) consisted of a $94 \times 94 \mathrm{~cm}$ piece of plywood, painted violet. Six white plastic drinking cups (Solo Co. No. P3A, Urbana, IL) were located as shown. These cups were $5.7 \mathrm{~cm}$ tall, $3.7 \mathrm{~cm}$ in diameter at the bottom, and $5.3 \mathrm{~cm}$ in diameter at the top. A red adhesive dot ( $1 \mathrm{~cm}$ in diameter) in the center of the bottom of each cup was covered by a small piece of clear tape. Drops of sucrose solu- 


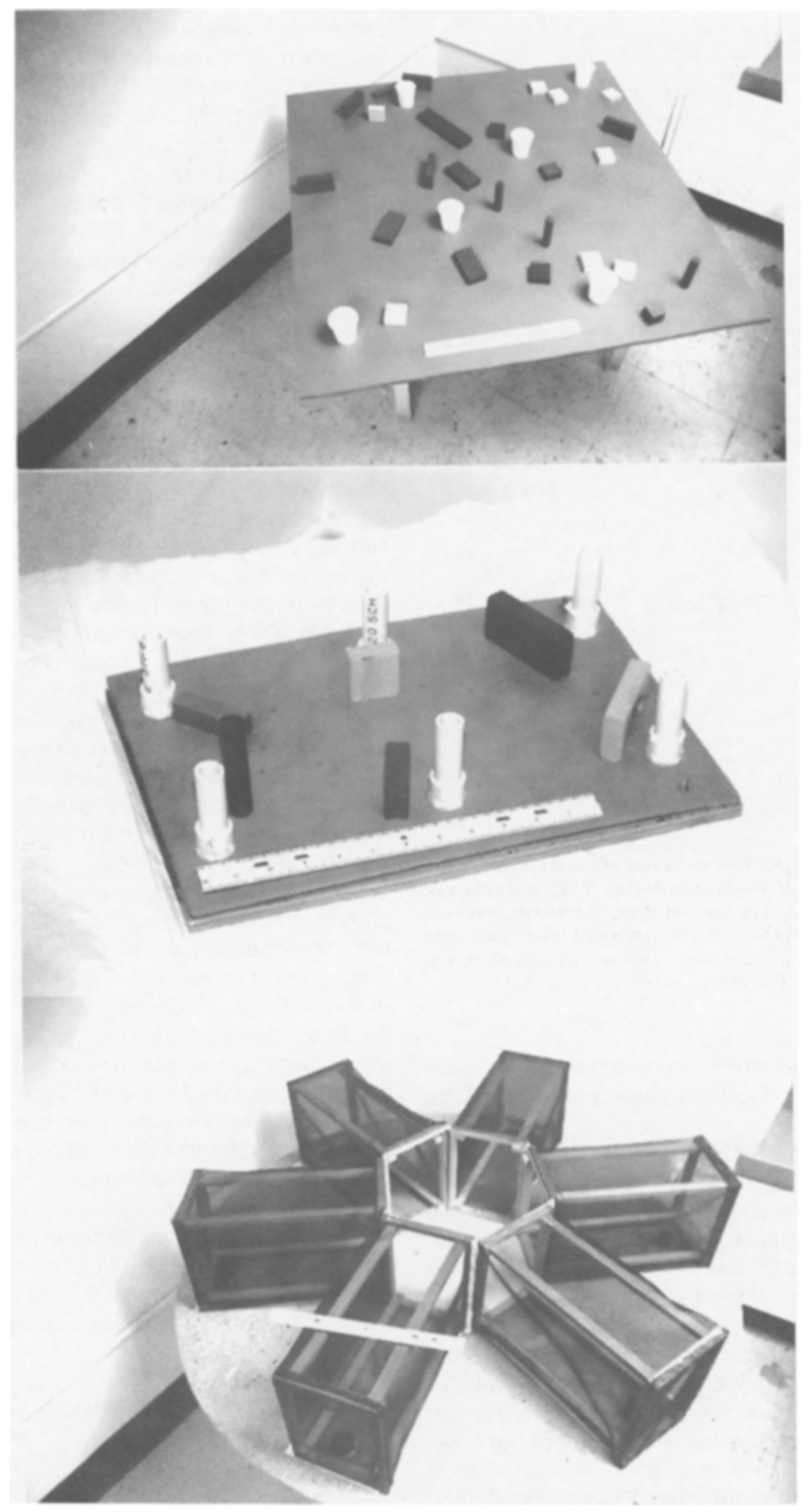

Figure 2. Apparatuses used in Experiments 2A (top panel), 2B (middle panel), and $2 \mathrm{C}$ (bottom panel). Ruler included to provide scale is $30.4 \mathrm{~cm}$ long. 
tion or water were placed on top of this dot (see Procedure). Cups were separated from their nearest neighbors by $35 \mathrm{~cm}$ (center to center). The apparatus surface included marks indicating cup locations so that cups could be easily removed and replaced. A set of children's wooden blocks, in a variety of shapes and colored red, blue, or yellow, were placed on the apparatus in the pattern shown in Figure 2 . These blocks were intended to serve as spatial landmarks. The apparatus was adjacent to, and at the same height as, the ledge of the window through which bees gained access to the laboratory.

The apparatus used in Experiment 2B (Figure 2, middle panel) consisted of a $42.5 \times 29 \mathrm{~cm}$ piece of plywood, painted violet. A $2 \times 3$ matrix of chambers was mounted on the plywood surface, with each chamber separated from its nearest neighbors by $18 \mathrm{~cm}$ (center to center). The chambers were constructed of white PVC plumbing fittıngs and tubing. A $1 / 2$-in. threaded fittıng formed the base of each chamber, into which a 6 -cm length of of $1 / 2$-in. tubing could be placed. The threaded portion of each fitting was inserted through a hole drilled in the apparatus surface. The resulting chamber had an interior diameter of $1.4 \mathrm{~cm}$ and was $9.5 \mathrm{~cm}$ in vertical length. A bee could enter the chamber at the top and crawl through the tube and fitting, which terminated on the surface of one the same plastic dishes used in Experiment 1, six of which were hidden underneath the apparatus (one at the terminus of each chamber). The entire apparatus could be easily lifted to allow these dishes and their contents to be manipulated (see Procedure). In addition, the tubing lengths forming the top portion of the chamber could be easily removed and replaced (see Procedure). Six children's blocks of various shapes and colors were mounted on the apparatus, as shown. The apparatus was placed on a window sill.

The apparatus used in Experiment $2 \mathrm{C}$ was a radial-arm maze (Olton \& Samuelson, 1976) with six arms (Figure 2, bottom panel). Each arm was constructed of a wooden frame covered with nylon mesh, and was $39 \mathrm{~cm}$ in length, $17 \mathrm{~cm}$ wide, and $22 \mathrm{~cm}$ tall. The arms were attached to a wooden floor, which was painted white. The central ends of the arms formed a six-sided arena, which was $31 \mathrm{~cm}$ across. A lid constructed of a wooden frame and nylon mesh could be used to enclose this central arena. Centered $5 \mathrm{~cm}$ from the end of each maze arm was a hole, into which was inserted a 3/4-in. threaded PVC plumbing fitting. These fittings were painted blue and had an interior diameter of $2.5 \mathrm{~cm}$ at the top and $1.8 \mathrm{~cm}$ at the bottom. Bees could crawl through these fittıngs and onto the surface of a plastic dish, mounted below the maze surface at the terminus of the fittıng. These plastic dishes could be easily removed and replaced (see Procedure). The maze was mounted on a table using a lazy Susan, allowing it to be easily rotated. The maze was adjacent to a laboratory window. Its surface was at approxımately the height of the window sill.

Procedure. The procedures used in these three experiments were the same except for the exceptions noted. These experiments were conducted simultaneously by three different experimenters. Each experıment was associated with one window of the building, through which bees flew to shuttle between the hive and the apparatus and near which the apparatus was located. These windows were located three floors below the hive, on different sides of the bulding.

The bee was brought to the laboratory in a small plastic vial. The vial was opened, turned upside down, and placed over a large drop of sucrose solution. This drop was located either in one of the cups serving as a baited location in the apparatus used in Experıment 2A, In one of the six chambers of the apparatus used in Experiment 2B (with the tube removed), or on a plastic dish in the central arena of the radial-arm maze (Experiment $2 \mathrm{C}$ ). Typically, several bees were exposed to this recruitment procedure simultaneously. Some of the bees so treated began imbibing the sucrose solution, at which point the vial was removed. The bee was allowed to feed to repletion, after which it flew out the window. Some of these bees later returned to the apparatus, at which time sucrose drops were avallable in the same or a similar location. Once a bee returned of its own accord, it was marked with a spot of paint on the thorax or abdomen (different colors being used to code bees in the three experiments). After a marked bee returned to the apparatus two or three tımes, it was considered a subject in the experiment, and the procedure described below was invoked.

Prior to each trial, each of the six locations was baited with a small (approximately 4 - to 5- $\mu \mathrm{l}$ ) drop of $50 \%$ sucrose solution using a syringe. When the bee flew in the window, the camcorder was act1vated to record behavior. The experimenter recorded the sequence of locations chosen until the trial was completed. A trial was defined as completed when (1) all six locations had been visited at least once, or (2) 5 min passed without a choice. A choice was defined when a bee touched the rim or inside of a cup corresponding to a location (Experiment 2A), the rim or inside of a PVC tube corresponding to a chamber (Experiment $2 \mathrm{~B}$ ), or the $\mathrm{PVC}$ connector at the end of a maze arm (Experiment $2 \mathrm{C}$ ). In Experiment $2 \mathrm{C}$, the central arena of the maze was covered with the lid during each trial.

After all six locations had been visited at least once, the exper1menter provided a large drop of sucrose solution to allow the bee to feed to repletion. In Experiment $2 \mathrm{~A}$, this large drop was placed in a cup identical to those used on the apparatus, but located on the window sill between the apparatus and the window. In Exper1ment $2 \mathrm{~B}$, the drop was in a plastic dish held adjacent to the top of the tube corresponding to the last choice made by the bee. The bee typically crawled onto the dish after emerging from the tube. In Experiment $2 \mathrm{C}$, the drop was in a dish placed in the central arena of the maze while the bee visited the last maze arm. In all three experıments, bees virtually always imbibed from this repletion drop and then flew out the window after completing a trial.

A procedure was used to eliminate any ability of bees to use an odor trail or "footprint pheromone" (Free \& Williams, 1983; Giurfa \& Núñez, 1992) as a discriminative cue in Experıments 2A and 2B. This procedure corresponded to that used in Experiment 1. During the v1sit following the initial visit to a particular location, the cup (Experiment 2A) or tube (i.e., the tube forming the top portion of each chamber in Experiment $2 B$ ) corresponding to that location was replaced with an identical cup or tube. In Experiment $2 \mathrm{~A}$, this replacement cup contained a drop of tap water (because the contents of the cup were potentially visible whıle choices were made in that experiment). As in Experiment 1, we did not attempt to replace replacement cups (or tubes) following third or subsequent visits to a location because the speed with which bees moved from location to location toward the end of trials (when most locations had been depleted of food) did not allow us to reliably perform the manipulation while the bee remained in a cup (or tube). Thus, any tendency to avoid a first revisit (i.e., a second visit) cannot be accounted for by an odor cue. However, once a bee revisited a location, an odor may have been present at that location.

Each bee was exposed to a series of such trials until 30 trials had been completed.

\section{Results}

The results were evaluated in terms of two measures of performance. The first was the number of locations revisited (one or more times) during the first six choices. This measure, developed in the context of Experiment 1, isolated performance which could be affected by odor traces possibly left after bees made second (or later) visits to locations in Experiments 2A and 2B. The second measure was the total number of choices required to visit all six locations. This measure was not used in Experiment I because bees in that experiment often did not visit all six locations. In the present experiment, all trials were 
completed by the bee visiting all six locations. An advantage of this measure is that it takes into account all choices made by the bees and therefore might be more sensitive to any tendency to avoid revisits. However, it is open to possible effects of odors or other physical traces that might be left by bees following the second visit to a particular location. Figure 3 shows the results of all three experiments in terms of both the mean number of locations revisited during the first six choices and the mean number of visits required to visit all six locations. In order to allow de-



Empirical Results
Estimates of Chance

Figure 3. Mean levels of choice accuracy in Experiments 2A, 2B, and 2C. Panels on the left show the mean number of locations that were revisited one or more times during the first six choices. Panels on the right show the mean number of visits required to visit all six locations. Data are shown in three blocks of 10 trials each. Two estimates of chance performance are shown. Estimates of chance performance based on the Monte Carlo simulations using the empirical transition probabilities $(M)$ are described in the text and were used in the statistical evaluations of choice accuracy. The strict estimates (S) are based on the assumption that bees chose randomly from among the six (first choice) or five (subsequent choices) available locations. They were determined in the same manner as the $M$ estimates, except that the simulation was not constrained by the empirical transition probabilities (instead, it chose randomly from among the alternatives). The strict estimate is considered to be a less accurate estimate of chance and is shown for illustrative purposes only. tection of changes in performance over the course of trials, the data were considered to be structured into three blocks of 10 trials each.

As in our earlier experiments and in Experiment 1, empirical data were compared with an estimate of the choice accuracy expected on the basis on the location-tolocation transition probabilities of each bee during each trial block. Each of these estimates was based on 1,000 iterations of a Monte Carlo simulation of bee choices based on the transition probabilities of individual bees. The transition probabilities in these experiments were similar to those found in Experiment 1 in that most bees appeared to show a moderate tendency to choose locations that were spatially proximal to the previously chosen location. It should also be noted that, as in all of the experiments reported in this paper, bees moved from location to location by flying rather than walking. As in Experiment 1 , immediate returns to locations were not included in the transition probabilities or counted in the empirical data because of the inherent ambiguity in determining whether these behaviors correspond to separate choices and because of the replacement of cups and tubes following the initial visit to each location (bees could not visit locations while we were in the process of replacing the cups or tubes in those locations). The estimates were compared to empirical performance using a within-subjects analysis of variance (ANOVA), which also included trial block as a factor.

The bees in Experiment 2A revisited fewer locations during the first six choices than indicated by the corresponding simulations $[F(1,19)=39.33]$. The magnitude of this difference did not significantly change over the course of trial blocks $[F(2,38)=2.98, p=.06]$. There was no evidence that bees made more correct choices during the first six choices than did the Monte Carlo simulations in Experiment 2B $[F(1,19)<1]$ or in Experiment $2 \mathrm{C}[F(1,15)=2.89]$. In terms of the number of choices required to complete each trial, the bees outperformed the Monte Carlo simulations in all three experiments [Experiment $2 \mathrm{~A}, F(1,19)=303.17$; Experiment $2 \mathrm{~B}$, $F(1,19)=34.86$; Experiment $2 \mathrm{C}, F(1,15)=26.73]$. The magnitude of the difference between empirical and simulated performance differed across trial blocks in Experiment $2 \mathrm{~A}[F(2,38)=4.53]$. There was no evidence for this interaction in Experiment $2 \mathrm{~B}[F(2,38)<1]$ or in Experiment $2 \mathrm{C}[F(2,30)=2.97]$.

\section{Discussion}

Bees in all three of these experiments revisited locations at which they had already depleted the sucrose solution less often than expected. This ability to avoid revisits cannot be attributed to systematic patterns or biases influencing the choice of location or the transitions between locations because of our technique of comparing performance with Monte Carlo simulations incorporating the location-to-location transition probabilities. Furthermore, this ability cannot be attributed to visual cues distinguishing baited from unbaited locations because such cues were not available in any of these experiments. 
A physical trace left by the bee, such as odor, could have contributed to this ability in Experiment 2C. In Experiments $2 \mathrm{~A}$ and $2 \mathrm{~B}$, however, our technique of replacing the cup or tube corresponding to a location following the first visit rules out such cues as an explanation for bees' ability to avoid the first revisit to a location. This ability is isolated by one of our performance measures (the number of locations revisited during the first six choices). Thus, in Experiment 2A, bees clearly demonstrated an ability to avoid revisits to locations, which cannot be explained in terms of physical traces left by the bee. Such traces remain possible as explanations for performance in Experiments 2B and 2C, although we have no evidence that they were employed by bees in those experiments.

The present results replicate those of Brown and Demas (1994; Demas \& Brown, 1995) and those of Experiment 1 under a wider variety of experimental conditions. The results therefore provide additional support for our earlier conclusion that honeybees possess spatial working memory. This working memory allows locations visited earlier in the trial to be discriminated from locations not yet visited.

However, in the variety of conditions included in the present experiment, the level of working memory ability exhibited by the bees remained limited. Although it is difficult to judge what constitutes sizable levels of working memory performance, by any account, the extent of such performance demonstrated by bees so far is small. For example, rats are typically found to visit approximately 7.5 novel locations during the first eight choices in a radial-arm maze and typically make fewer than two errors in completing an eight-arm maze. In contrast, bees in the present experiment made between four and eight errors in visiting six locations.

\section{EXPERIMENT 3}

Clearly, there are a number of unexamined features of our apparatus or procedure that might limit the extent of working memory ability demonstrated by bees. One approach to understanding the small size of the working memory effects we have obtained so far is to examine some potential constraints on working memory performance. One possibility is that there is a restriction on the ability of honeybees to discriminate among the locations involved in the experiments we have conducted.

The present experiment examined this possibility using a task that required the bees to discriminate among locations identical to those used in Experiments 2A and 2B. Specifically, for each bee there were two locations that were never baited, with the remaining four locations baited prior to each trial as in the earlier experiments. Bees were thus encouraged to avoid any visits to these two never-baited locations, and their ability to do so was measured. This task can be conceptualized as a referencememory task (Honig, 1978) in that the identity of these never-baited locations remains constant throughout the experience of the bee. It is also very similar to a number of discrimination learning tasks that have been used with bees by a number of researchers.

Bees have demonstrated high levels of visual and spatial discrimination ability in both field and laboratory studies (see, e.g., Cartwright \& Collett, 1982; Dyer, 1994; Gould, 1987; Huber, Couvillon, \& Bitterman, 1994; Menzel, Erber, \& Masuhr, 1974; Zhang, Bartsch, \& Srinivasan, 1996). The present experiment was intended to reveal whether similarly high levels of discrimination ability could be found in the context of the locations used in our working memory experiments. If the level of working memory ability found in our experiments is limited by a poor ability of bees to discriminate among the six locations used in those experiments, then bees should be able to learn to avoid visits to the never-baited location only at low levels of accuracy. On the other hand, if the low levels of working memory ability we have reported are due to limitations of working memory itself, then high levels of discrimination ability are possible.

\section{Method}

Subjects. The subjects in this experiment were from the same source and were transported to the laboratory in the same manner as in Experiments 1 and 2. Twelve and 11 subjects participated in Experiments $3 \mathrm{~A}$ and $3 \mathrm{~B}$, respectively.

Apparatus. The apparatuses used in these experiments were the same as those used in Experiment 2; that used in Experiment 3A was the same as that used in Experiment $2 \mathrm{~A}$, and that used in Experiment $3 \mathrm{~B}$ was the same as that used in Experıment $2 \mathrm{~B}$.

Procedure. The procedures used in these experiments were the same as those used in the corresponding subexperiment of Experiment 2, with the exceptions noted below. Prior to each trial of the experiment, four of the six cups (Experiment $3 \mathrm{~A}$ ) or chambers (Experiment 3B) were baited. The two never-baited locations were determined randomly for each bee with the restriction that the identity of never-baited locations was distributed as equally as possible across the six locations over bees (each location was assigned as a never-baited location for 4 bees in Experıment $3 \mathrm{~A}$ and for either 3 or 4 bees in Experiment 3B). The never-baited locations were determined prior to the first trial for each bee, and they remained constant over the course of the experiment. A trial was considered complete when a bee had visited all four baited locations. In Experıment $3 \mathrm{~A}$, a cup in a never-baited location contained a drop of tap water, and cups were replaced with a fresh cup containing a drop of tap water following the first visit to the cup. In Experiment 3B, the tube forming the top portion of a chamber was replaced with a fresh tube following the first visit to the chamber. Each bee completed 30 trials.

\section{Results}

To assess the acquisition of any ability to avoid visits to the never-baited locations, data from the 30 trials of each bee were considered to be structured into six blocks of five trials each. To isolate the ability of bees to avoid visits to never-baited locations from the working memory ability used to avoid revisits to locations, only initial visits to locations made during the first four choices of each trial were evaluated. Figure 4 shows the mean percentage of these choices that occurred to the never-baited locations in Experiment 3A (top panel) and Experiment 3B (bottom panel). Also shown is the a priori estimate (33.3\%) of visits directed toward never-baited locations, assum- 

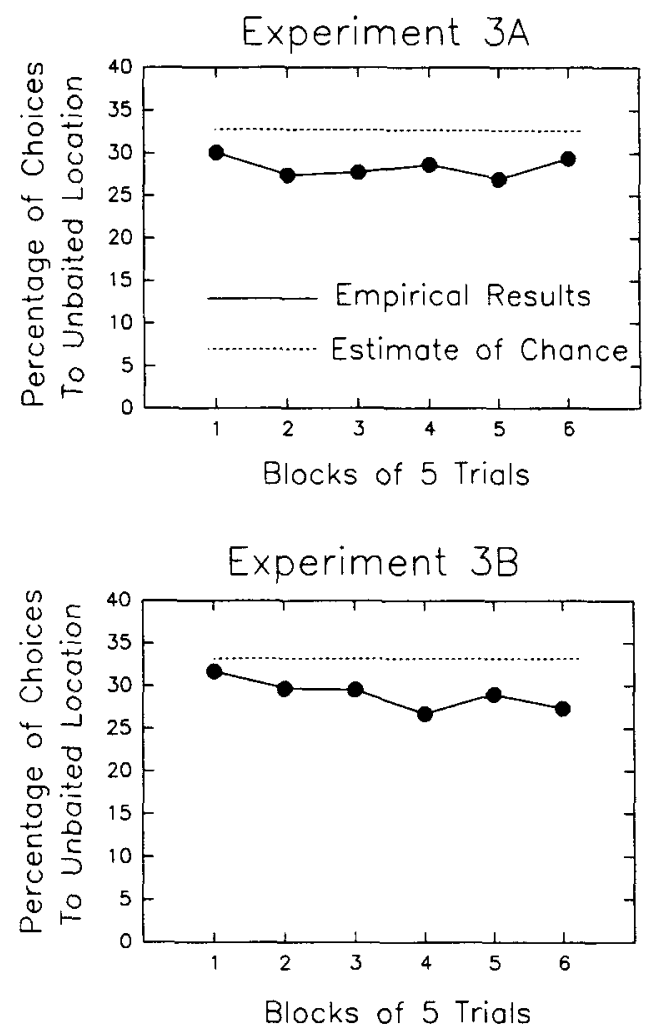

Figure 4. The mean percentage of choices that were to the unbaited locations and the percentage expected on the basis of chance in Experiments $3 A$ and $3 B$.

ing that bees are insensitive to the identity of neverbaited locations.

There was no evidence that the percentage of choices directed to never-baited locations changed over the course of trial blocks in Experiment $3 \mathrm{~A}[F(5,55)<1]$ or Experiment $3 \mathrm{~B}[F(5,50)<1]$. The percentage of choices directed to never-baited locations, collapsed over trial blocks, was reliably less than $33.3 \%$ in Experiment $3 \mathrm{~A}[t(11)=1.8]$ and in Experiment $3 \mathrm{~B}[t(10)=2.5]$.

\section{Discussion}

Although bees in these two experiments did demonstrate a reliable tendency to avoid visits to never-baited locations, the magnitude of this tendency was quite small and did not increase over the course of the experiment. This is surprising given bees' ability to learn to visit a baited target rather than an unbaited target using a variety of cues, including spatial location in relation to proximal or distal landmarks (Cartwright \& Collett, 1982; Huber et al., 1994), color (Menzel et al., 1974), and shape (Gould, 1987), all of which were available as discriminative cues in the present experiments.

For example, Gould (1987) provided bees with 10 training visits in which food was available between two identical landmarks and then tested them using a threealternative choice test in which the correct location was between the training landmarks, and the incorrect loca- tions were proximal to landmarks that differed in shape from the training landmark. Depending on the nature of the shape difference, bees chose the correct location with $60 \%-90 \%$ accuracy (with $33 \%$ being expected by chance). Similarly, Gould found that bees learned to choose one location among three on the basis of the color of landmarks with a choice accuracy of approximately $90 \%$ (again, chance is $33 \%$ ). Similarly, Couvillon, Bitterman, and their colleagues have consistently found that bees can learn to choose nearly perfectly between two targets on the basis of color or odor within 10 trials (see Couvillon \& Bitterman, 1991, for a review). The work of these and other researchers clearly indicates that honeybees are capable of much higher levels of choice accuracy than were found in the present experiment.

Furthermore, the details of some of these previous experiments suggest that the specific discriminative cues provided in the present experiments should have been adequate to support higher levels of discrimination ability than was found. In Gould's (1987) experiments, for example, locations were arranged in a linear pattern, separated by $30 \mathrm{~cm}$. This spatial separation is similar to that of Experiments $2 \mathrm{~A}$ and $3 \mathrm{~A}$, and the shapes and colors we used are also similar to Gould's. Huber et al. (1994) found high levels of discrimination accuracy when a baited target and an unbaited target were separated by $40 \mathrm{~cm}$ (with or without the provision of a proximal landmark) or by $10 \mathrm{~cm}$ (only when a proximal landmark was provided). Thus, it seems unlikely that discrimination ability in the experiment was limited by the nature of the spatial cues or spatial separations among the target locations.

One possible explanation for the relatively low levels of discrimination ability involves the fact that multiple baited targets (four) were available in the present experiments, and they had to be discriminated from multiple unbaited targets (two). Experiments in which bees have demonstrated high levels of discrimination ability have involved a single target location or stimulus, which is discriminated from one or more distractor locations or stimuli. It is possible that the existence of multiple targets limits the discrimination performance of bees. A second possible explanation is suggested by the fact that "baited" targets in the present experiment provided reinforcement only during the first visit, and were therefore similar to the unbaited locations (in that they did not contain food) during any subsequent visits. This partial reinforcement of positive targets may have resulted in lowered levels of discrimination performance.

We cannot determine the explanation for the limited extent of discrimination ability exhibited by our bees. However, its existence can help explain the low levels of working memory performance found in Experiments 1 and 2. Specifically, working memory performance would be expected to be constrained by any limitation in the ability of subjects to discriminate among the items coded in working memory. The low levels of working memory ability found in our earlier experiments could be explained by a limited ability to discriminate among the six locations. The results of the present experiments, which did 
not involve working memory but did involve discrimination among the locations, are entirely consistent with this possibility.

\section{GENERAL DISCUSSION}

The results of four experiments $(1,2 \mathrm{~A}, 2 \mathrm{~B}$, and 2C) replicate our earlier findings (Brown \& Demas, 1994; Demas \& Brown, 1995) that bees avoid revisits to locations that have been depleted of sucrose solution. In three cases (Experiments 1, 2A, and 2B), an analysis that examines a subset of the bees' choices provides a means of ruling out odor or other subject-generated cues as an explanation for this ability. This is important given that odor left by bees on visited locations has been implicated as providing a cue in both natural (Free \& Williams, 1983) and laboratory (Giurfa \& Núñez, 1992) foraging situations. In two of these three cases (Experiments 1 and 2A), clear evidence for an ability to avoid revisits that cannot be explained by such odor trails was found. Because our estimate of chance accounts for location-to-location movement biases of individual bees, the tendencies to avoid revisiting locations reported in this paper cannot be explained in terms of systematicity or stereotypy in the movement of the bees among locations. Furthermore, our experimental procedures are not open to the criticisms of our earlier experiments offered by Burmeister et al. (1995). Thus, these data demonstrate convincingly that bees avoid revisits to locations on the basis of memories of the identity of locations visited earlier in the trial.

A word is in order regarding the Monte Carlo simulations used to estimate the level of choice accuracy expected on the basis of chance in Experiments 1 and 2 and in some of our previous experiments (Brown \& Demas, 1994, Experiment 1; Demas \& Brown, 1995). In each case, the location-to-location transition probabilities of individual bees (e.g., those represented by the means in Table 1) determine the choices of the simulation. Critics have suggested that a better estimate of chance performance would include transition probabilities conditionalized not just on the immediately preceding choice, but on choices made earlier in the choice sequence. For example, bees might have a tendency to move in a straight line across rows of baited locations, and our estimate of chance would not fully represent this tendency. By definition, however, such a tendency requires that the bee's next choice be contingent on a previous choice. The identity of that previous choice (or something corresponding to it) is therefore a memory. The first-order transition probabilities are included in our estimate of chance because the location of choice $n-1$ serves as a stimulus that could very well determine the direction of flight and therefore the identity of choice $n$. But any pattern of choices in which the next location chosen depends on choices made in the past (and therefore not currently perceived) meets any reasonable criterion for the involvement of memory. It should also be mentioned that Brown and Demas performed two experiments that did not involve the use of a Monte Carlo simulation in the estimation of chance performance (Brown \& Demas, 1994, Experiments 2 and $3)$. The results of these experiments supported the conclusions on the basis of the Monte Carlo simulations and therefore provide independent, converging evidence for the involvement of memory in honeybee spatial choice.

In order to allow previously visited locations to be discriminated from those not yet visited, the contents of this memory must change during the course of each trial and between trials. Thus, this memory appears to meet the defining criteria that are used to identify the working memory ability that has been studied in vertebrates (Baddeley, 1986; Honig, 1978; Sherry \& Schacter, 1987). Demas and Brown (1995) showed that the contents of this memory can be used in a flexible manner, allowing bees to either avoid additional visits (win-shift, as in the present experiments) or selectively revisit locations (win-stay). In the latter condition of Demas and Brown's experiment, bees found sucrose solution only in those locations that they had previously visited, and the bees were found to selectively visit those locations. Thus, the memory system inferred on the basis of our results cannot be understood in terms of associative learning, with associative strength corresponding to each location and being modified by the outcome of visits (see, e.g., Greggers \& Menzel, 1993). Such a view predicts that future responding to a location be determined by the outcome of previous visits to that location. But, in Demas and Brown's experiment, a reinforced visit to a location could result in either an increase in the tendency to visit that location (win-stay) or a decrease in that tendency (win-stay). These considerations lend further support to the interpretation of these results in terms of a working memory system, the contents of which are used by other systems to determine behavior.

The working memory ability revealed in our experiments may or may not be related to the bee short-term memory elegantly demonstrated by the work of Menzel $(1979,1983,1990)$. He and his colleagues have provided evidence for at least two different physiological processes involved in the formation and consolidation of memories in honeybees. These processes have different time courses, with short-term memory having a duration of approximately $30 \mathrm{sec}$. The function of this short-term memory system as described by Menzel is to provide information to a longer term memory that corresponds to each stimulus and controls responding to that stimulus. Working memory, on the other hand, is conceived of as holding a limited amount of information being used in the context of ongoing cognitive processes or behaviors (see, e.g., Honig, 1978). In the case of spatial working memory, this information is a set of locations, such as the locations that have been previously visited within a particular trial of Experiment 1 or 2 (see also Olton, 1978). Because the identity of visited locations changes both within each trial and from one trial to the next, the content of working memory must change in a dynamic and flexible manner.

Although the present data convincingly demonstrate the existence of working memory in bees, they are equally clear that the extent of the working memory performance 
exhibited in our experiments is limited. ${ }^{1}$ Experiment 2 was intended to provide a range of experimental conditions that might support higher levels of spatial working memory performance than we had previously found. Bees demonstrated an ability to avoid revisits under this range of conditions, but the extent of that ability was consistently low relative to the levels of spatial working memory performance that has been demonstrated by rats and a variety of other vertebrates.

It may be that the relatively low levels of spatial working memory performance found in these experiments is a reflection of relatively impoverished spatial working memory ability in bees. However, the results of Experiment 3 suggest a different interpretation. For reasons that cannot be determined on the basis of the present results, honeybees appear to have difficulty discriminating among the locations used in the present experiments. Thus, an accurate determination of the extent of spatial working memory in bees will require additional work in which bees choose from among locations (or other response alternatives) that can be shown to support high levels of discrimination ability.

\section{REFERENCES}

ABRamson, C. I. (1994). A primer of invertebrate learning. The behavioral perspective. Washington, DC: American Psychological Association. Baddeley, A. (1986). Working memory. Oxford: Oxford University Press.

Bitterman, M. E. (1996). Comparative analysis of learning in honeybees. Animal Learning \& Behavior, 24, 123-141.

BRown, M. F. (1993). Sequential and simultaneous choice processes in the radial-arm maze. In T Zentall (Ed.), Animal cognition: $A$ tribute to Donald A Riley (pp. 153-173). Hillsdale, NJ: Erlbaum.

Brown, M. F., \& Demas, G. E. (1994). Evidence for spatial working memory in honeybees (Apis mellifera). Journal of Comparative Psychology, 108, 344-352.

Brown, M. F., \& HUGGINs, C. K. (1993). Maze-arm length affects a choice criterion in the radial-arm maze Animal Learning \& Behavior, 21, 68-72.

Brown, M. F., \& Lesniak-Karpiak, K. B. (1993). Choice criterion effects in the radial-arm maze: Maze-arm incline and brightness. Learning \& Motivation, 24, 23-39.

Burmeister, S., Couvillon, P. A., \& Bitterman, M. E. (1995). Performance of honeybees in analogues of the rodent radial maze. Animal Learning \& Behavior, 23, 369-375.

Cartwright, B. A., \& Col.letr, T. S. (1982). How honeybees use landmarks to guide their return to a food source. Nature, 295, 560-564.

Corbet, S. A., Kerslake, C. J. C., Brown, D , \& Morland, N. E. (1984). Can bees select nectar-rich flowers in a patch? Journal of Apicultural Research, 23, 234-242.

Couvillon, P. A., \& Bitterman, M. E. (1991). How honeybees make choices. In L. J. Goodman \& R. C. Fisher (Eds.), The behavior and physiology of bees (pp. 116-130). Wallıngford, U.K.: CAB International.

Demas, G. E., \& BRown, M. F. (1995). Honeybees are predisposed to win-shift but can learn to win-stay. Animal Behaviour, 50, 1041-1045.

DYER, F. C. (1994). Spatial cognition and navigation in insects. In L A. Real (Ed.), Behavioral Mechanisms in Evolutionary Ecology (pp. 6698). Chicago: University of Chicago Press.

Free, J B., \& Williams, I. H. (1983) Scent-marking of flowers by honeybees. Journal of Apicultural Research, 22, 86-90.

GiURFA, M., \& NúÑ̃E, J. A. (1992). Honeybees mark with scent and reject visited flowers. Oecologia, 89, 113-117.

GouLD, J. L. (1987). Landmark learning by honeybees. Animal Behaviour, 35, 26-34.
GregGers, U., \& MENZEL, R. (1993). Memory dynamics and foraging strategies of honeybees. Behavioral Ecology \& Sociobiology, 32, $17-29$.

HoNig, W. K. (1978). Studies of working memory in the pigeon. In S. H. Hulse, H. Fowler, \& W. K. Honig (Eds.), Cognitive processes in animal behavior (pp. 211-248). Hillsdale, NJ. Erlbaum

Huber, B., Couvillon, P. A., \& Bitterman, M. E. (1994). Place and position learning in honeybees (Apis mellifera). Journal of Comparative Psychology, 108, 213-219.

Isnec, M. R., Couvillon, P. A., \& Bitterman, M. E. (1997). Shortterm spatial memory in honeybees. Animal Learning \& Behavior, 25, 165-170.

Kamil, A. C., Balda, R. P., \& Olson, D. J. (1994). Performance of four seed-caching corvid species in the radial-arm maze analog. Journal of Comparative Psychology, 108, 385-393.

MenzeL, R. (1979). Behavioural access to short-term memory in bees. Nature, 281, 368-369.

MENZEL, R. (1983). Neurobiology of learning and memory: The honeybee as a model system. Naturwissenschaften, 70, 504-511.

Menzel, R. (1990). Learning, memory and "cognition" in honeybees. In R. P. Kesner \& D. S. Olton (Eds.), Neurobiology of comparative cognition (pp. 237-292). Hillsdale, NJ: Erlbaum.

Menzel, R., Erber, J., \& Masuhr, T. (1974), Learnıng and memory in the honeybee. In L. Barton-Browne (Ed.), Experimental analysis of insect behavior (pp. 195-217). Berlin: Sprınger-Verlag.

Ohyama, T., Couvillon, P. A., \& Bitterman, M. E. (1995). Perseveration in the color choices of honeybees. Journal of Insect Behavior, $8,409-415$.

Olton, D. S. (1978) Characteristics of spatial memory. In S. H. Hulse, H. Fowler, \& W. K. Honig (Eds.), Cognitive processes in animal behavior (pp. 341-373). Hillsdale, NJ: Eribaum.

OLTON, D. S., \& SAMUELSON, R. J. (1976). Remembrance of places past: Spatial memory in rats. Journal of Experimental Psychology'Animal Behavior Processes, 2, 97-116.

PAPAJ, D. R., \& LEWIS, A. C. (1993). Insect learning: Ecological \& evolutionary perspectives. New York: Chapman \& Hall.

RoBerTS, W. A., \& Grant, D. S. (1974). Short-term memory in the p1geon with presentation time precisely controlled. Learning \& Memorn, 5, 393-408.

ROBERTS, W. A., \& VAN VELDHUIZEN, N. (1985). Spatial memory in p1geons on the radial maze. Journal of Experimental Psychology: Animal Behavior Processes, 11, 241-260.

SHERRY, D. F., \& SCHACTER, D. L. (1987). The evolution of multiple memory systems. Psychological Review, 94, 439-454.

WetherwaX, P. B. (1986). Why do honeybees reject certain flowers? Oecologia, 69, 567-570.

Zhang, S. W., Bartsch, K., \& Srinivasan, M. V. (1996). Maze learning by honeybees. Neurobiology of Learning \& Memory, 66, 267-282.

\section{NOTE}

1 In interpreting the level of performance exhibited by the bees, it should be kept in mind that cases in which bees left a location and then immediately returned to the same location (with no visits to other locations intervening) were not considered in the analysis. This is because it is not clear what constitutes a unit of choice in this circumstance and also because of the possibility that switching dishes, cups, and tubes (in Experıments $1,2 \mathrm{~A}$, and $2 \mathrm{~B}$, respectively) could affect the tendency to immediately return to the just-chosen location. Such immediate revisits were not included in the empirical results, and so the transition matrices used by the simulations never produced such immediate revisits in the estimates of expected performance. However, if working memory contributes to a tendency to avold immediate returns to locations, then our measures underestimate the levels of working memory performance. Despite this consideration, however, the extent of working memory performance found in these experiments is quite small.

(Manuscript received January 13, 1997, revision accepted for publication May 14, 1997.) 